\title{
Light Ion Yields from Bombardment of Thick Targets by Protons, Helium-4 and Iron-56
}

\author{
Luis A. Castellanos ${ }^{1 *}$, Natalie A. McGirl ${ }^{1}$, Ashwin P. Srikrishna ${ }^{l}$, Lawrence H. Heilbronn ${ }^{l}$, Chiara La Tessa ${ }^{2}$, Adam $^{2}$ \\ Rusek ${ }^{2}$, Michael Sivertz ${ }^{2}$, Steve Blattnig ${ }^{3}$, Martha Clowdsley ${ }^{3}$, Tony Slaba ${ }^{3}$ and Cary Zeitlin ${ }^{4}$ \\ ${ }^{1}$ Department of Nuclear Engineering, University of Tennessee, 1004 Estabrook Rd, Knoxville, TN, USA, 37996 \\ ${ }^{2}$ NASA Space Radiation Laboratory, Brookhaven National Laboratory, Upton, NY, USA, 11973 \\ ${ }^{3}$ National Aeronautics and Space Administration, Langley Research Center, Hampton, VA, USA, 23681 \\ ${ }^{4}$ Lockheed Martin, Information Systems and Global Solutions, Houston, TX, USA, 77285
}

\begin{abstract}
In March 2016 accelerator-based experiments colliding protons ( 0.4 and $0.8 \mathrm{GeV}$ ), helium (0.4 AGeV) and iron (0.4 and 0.8 AGeV) on thick aluminum targets with surface densities of 20, 40, and $60 \mathrm{~g} / \mathrm{cm} 2$ were performed at the National Aeronautics and Space Administration Space Radiation Laboratory (NSRL) at Brookhaven National Laboratory. Two targets were utilized in each experimental configuration. Hydrogen and helium ions were detected using organic liquid scintillators in conjunction with thin plastic scintillators at $10^{\circ}, 30^{\circ}, 45^{\circ}, 60^{\circ}, 80^{\circ}$, and $135^{\circ}$ from beam axis. Time-of-flight techniques and pulse shape discrimination were used to identify light ion species in order to generate double differential energy spectra of the light ion fragments. Comparisons of these measured yields were compared with Monte Carlo calculations generated by MCNP6. These yields will be used to quantify uncertainty in radiation transport codes utilized in risk assessment for spaceflight missions with prolonged exposures to galactic cosmic rays.
\end{abstract}

\section{Introduction}

The radiation environment beyond the confines of Earth's magnetosphere poses a significant risk for future manned missions. While exposure from Solar Energetic Particles (SEP) can be mitigated with increased shielding, the Galactic Cosmic Ray (GCR) component of dose is pervasive. Current calculations of Radiation Exposure Induced Death (REID) suggest that increased shielding has a negligible ability to reduce cancer risks [1]. These estimates of risk are based on particle fluences simulated by deterministic radiation transport codes, which are verified and validated by fits to measurements of GCR ion fluences from probes in Earth's atmosphere. The available database for comparison is limited, as the majority of data were collected at energies below $500 \mathrm{AMeV}$ [2]. However, GCR at these energies are limited contributors to effective dose [3]. The limitations of the available data increase the uncertainties associated with transport calculations and subsequent risk assessments from GCR ions at higher energies. The purpose of the experiment is to provide measurements of double differential light ions yields through thick targets composed of materials commonly selected to shield against GCR. This information will be used to further benchmark transport codes at energies that are inadequately represented by prior measurement efforts.

\section{Experiment Overview}

Three separate ion species were delivered to the National Aeronautics and Space Administration (NASA) Space Radiation Laboratory (NSRL) target room over a 100hour timespan from the Booster accelerator at Brookhaven National Laboratory (BNL). H-1, He-4, and $\mathrm{Fe}-56$ beams were extracted at $0.4 \mathrm{AGeV}$, while $\mathrm{H}-1$ and $\mathrm{Fe}-56$ were extracted at $0.8 \mathrm{AGeV}$,. These projectiles were incident on the the center of the primary upstream target, composed of either aluminum with thicknesses of 20,40 , and $60 \mathrm{~g} / \mathrm{cm}^{2}$ and area $30 \mathrm{x} 100 \mathrm{~cm}^{2}$. A second downstream target was placed $3.5 \mathrm{~m}$ from the center of the upstream target. This configuration mirrors an enclosed space which was studied in work that identifies a local minimum in dose equivalent as a function of shielding thickness [4]. The downstream target matched the material of the upstream target with area $100 \times 100$ $\mathrm{cm}^{2}$ and a fixed $60 \mathrm{~g} / \mathrm{cm}^{2}$ thickness, regardless of the upstream target's thickness.

Secondary light ions produced in the upstream target were detected with six cylindrical EJ-301 organic scintillator detectors, with active regions of dimensions 12.7- $\mathrm{cm}$ diameter and depth. In front of each detector was a thin NE-304 plastic scintillator to discriminate, or veto, charged and uncharged particles. The detectors were placed at $10^{\circ}, 30^{\circ}, 45^{\circ}, 60^{\circ}, 80^{\circ}$, and $135^{\circ}$ from beam axis,

*Corresponding author: lcastell@vols.utk.edu 
as illustrated in Figure 1. Total measured particle yields decreased with increasing angle and distance from target center. This influenced the choice of detector distances from target center, with care not to saturate the detection system and to accommodate shadow bars for neutron measurement. Timing information was initiated with a coincidence event between two thin EJ-228 plastic scintillators upstream from the primary target.

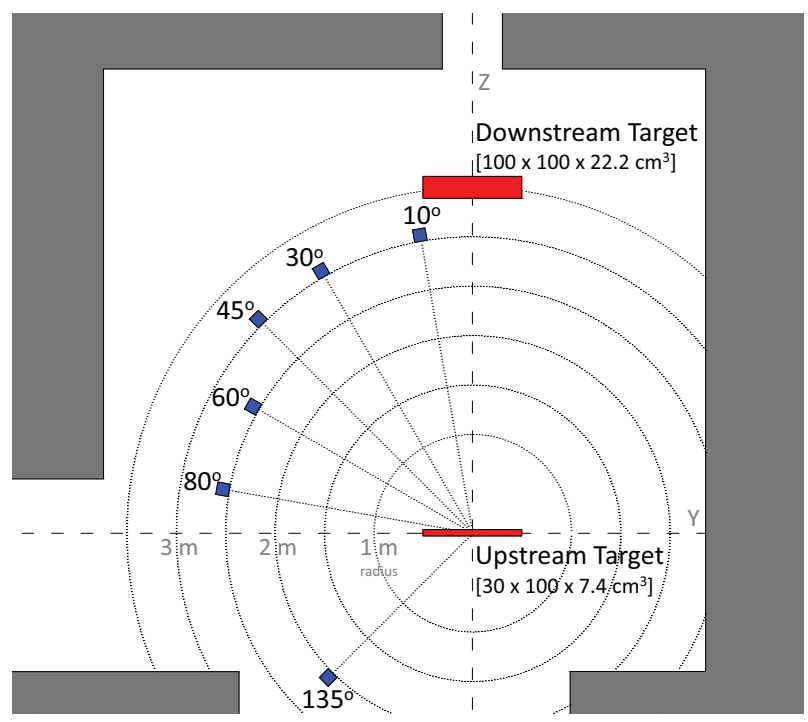

Fig. 1. NSRL target room schematic with experiment equipment illustrated, $20 \mathrm{~g} / \mathrm{cm} 2$ target shown.

This investigation will continue the efforts of the 18hour commissioning experiment conducted in May 2015. Additional measurements will be conducted at NSRL to examine the yields resulting from other projectile species and energies, as well as other target materials and compositions in November 2016 and 2017.

\section{Data Analysis Methodology}

Light ions are identified by comparison of velocity and energy deposited in the detector. Some ions deposit their full kinetic energies in the detector. By interpolating from the continual slowing down approximation range tables provided by NIST [5], the full detector length of $12.7 \mathrm{~cm}$ is enough material to stop a proton of approximately 121.5 $\mathrm{MeV}$. Particles with higher energies can escape or "punch through" the detector. The range $(R)$ of a proton is related to the range of a different projectile $\left(R_{i}\right)$ of mass $(A)$ and charge number $(Z)$ with the same velocity in an absorber of density $(\rho)$ by equation 1 . This relation is the key for determining the velocities of other ions

$$
(R \rho)_{i}=\left(A / Z^{2}\right)_{i} \cdot(R \rho)_{\text {proton }}
$$

An example of a histogram of light ion time of flight (ToF) and energy deposited (E) is presented in Figure 2. Detection events pertaining to the same ion are illustrated as pairs of lines which join at a peak. Peaks are indicative of particles losing full energies in the detector, with the line to the right of these peaks indicative of ions punching through the detection volume. Ions with higher mass deposit more energy and subsequently populate the upper parts of the histogram. The three lower pairs of lines represent hydrogen ions, the rightmost depicts proton detection events. This technique allows for discrimination between different secondary ion species' ToF spectra, which are next converted to energy spectra using the known distance between detector and the target.

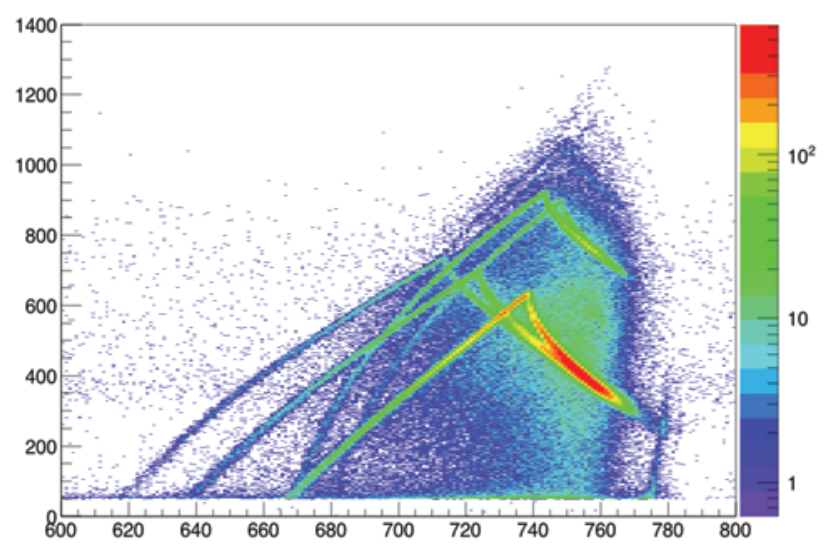

Fig. 2. Total energy deposited (Y) vs time-of-flight (X) histogram for $\mathrm{Fe} 0.4-\mathrm{AGeV}$ projectiles at $10^{\circ}$.

Further discernment between particles was achieved utilizing pulse shape discrimination (PSD) techniques. For histograms, such as Figure 2, illustrating the detection of multiple ions and isotopes, there are points where the respective ions' charge deposition signatures overlap. PSD utilizes a feature of the data acquisition apparatus which integrates the charge deposition in two ways. One integrates the signal over the full 200-nanosecond "live" time of the acquisition system for a given detection event. The other is to integrate only the first 20 nanoseconds of the signal. Different ions produce signals in the detectors with different rise and fall times, and this feature of the scintillation allows for these two integration modes to serve as a tool for discerning differing ions from each other. A histogram charting these integrated values for the ions detected at the $10^{\circ}$ position resulting from a 0.4 $\mathrm{AGeV}$ projectile is presented in Figure 3. The diagonal yellow line at the top right of the figure is indicative of helium ions. The two diagonal red-yellow bands at the lower left of the figure are indicative of protons, the lower of the two representative of protons depositing their full kinetic energies and while those populating the other line have enough energy to punch through. The ability to select events on this histogram allows one to most accurately attribute detection events to the correct ion at points where lines cross in the charge deposition vs ToF histograms. 


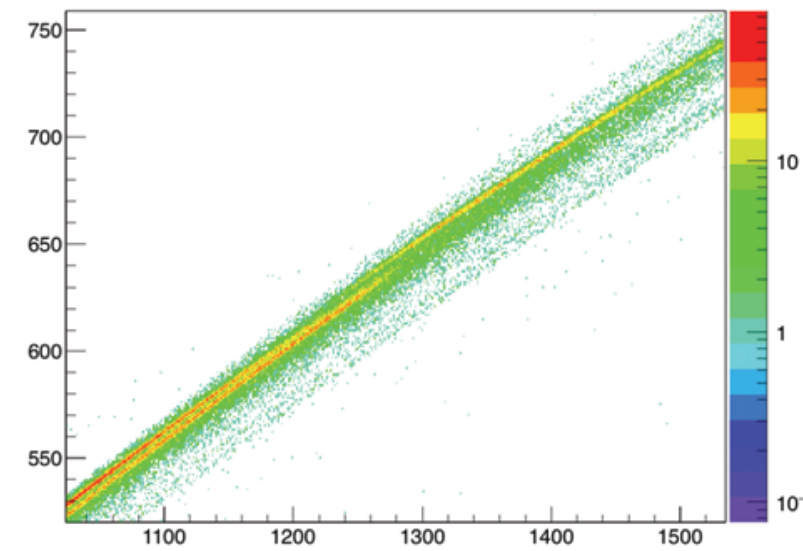

Fig. 3. Charge deposited in the first $20 \mathrm{~ns}$ of signal (Y) vs. total charge deposited (X) over the full 200ns integration gate.

\section{Experiment Results}

A summary of secondary ions detected organized by incident projectile is presented in Table 1. The angles listed are the widest angle of detection, thus detectors positioned at shallower or smaller angles than what is listed were also able to detect that respective ion. It was expected to not detect the heavier mass number greater than two ions from bombardments with proton projectiles.
Table 1. Summary of secondary ions detected resulting from bombardments incident projectiles and widest position of detection.

\begin{tabular}{|l|l|l|l|l|l|}
\hline Projectile & H-1 & H-2 & H-3 & He-3 & He-4 \\
\hline $\begin{array}{l}0.4- \\
\text { AGeV Fe }\end{array}$ & $135^{\circ}$ & $80^{\circ}$ & $45^{\circ}$ & $30^{\circ}$ & $30^{\circ}$ \\
\hline $\begin{array}{l}0.8- \\
\text { AGeV Fe }\end{array}$ & $135^{\circ}$ & $80^{\circ}$ & $60^{\circ}$ & $30^{\circ}$ & $30^{\circ}$ \\
\hline $\begin{array}{l}0.4-G e V \\
\text { H }\end{array}$ & $135^{\circ}$ & $45^{\circ}$ & - & - & - \\
\hline $\begin{array}{l}0.8-G e V \\
\mathrm{H}\end{array}$ & $135^{\circ}$ & $60^{\circ}$ & - & - & - \\
\hline $\begin{array}{l}0.4- \\
\text { AGeV He }\end{array}$ & $135^{\circ}$ & $60^{\circ}$ & $45^{\circ}$ & $30^{\circ}$ & $30^{\circ}$ \\
\hline
\end{tabular}

A small sample of the results collected will be presented in this paper to maintain brevity, while still showcasing the comprehensive detection capabilities of the experiment apparatus. Double differential yields of protons stemming from $0.4-\mathrm{AGeV}$ iron on the three thicknesses of aluminium used are presented in Figure 4. The results exhibited predictable trends of yield as a function of angle. The relative rates of production per ion were also approximately what were expected, as supported in comparisons of the measured results to those simulated using MCNP6 [6]. An example of this comparison is presented in Figure 5.
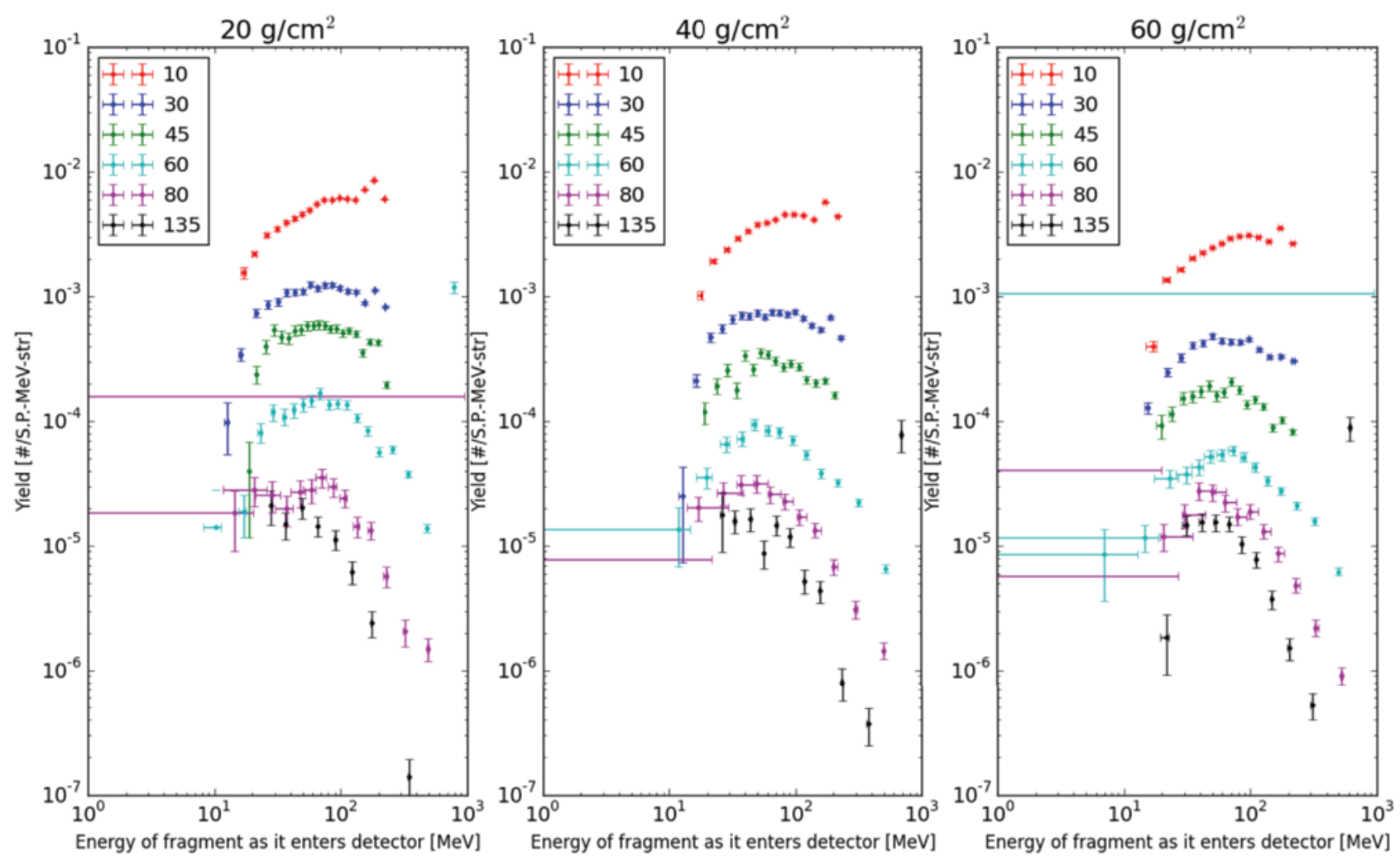

Fig. 4. Measured yields of protons resulting from the bombardment of aluminum upstream targets of three separate thicknesses by $0.4-\mathrm{AGeV}$ Fe. 

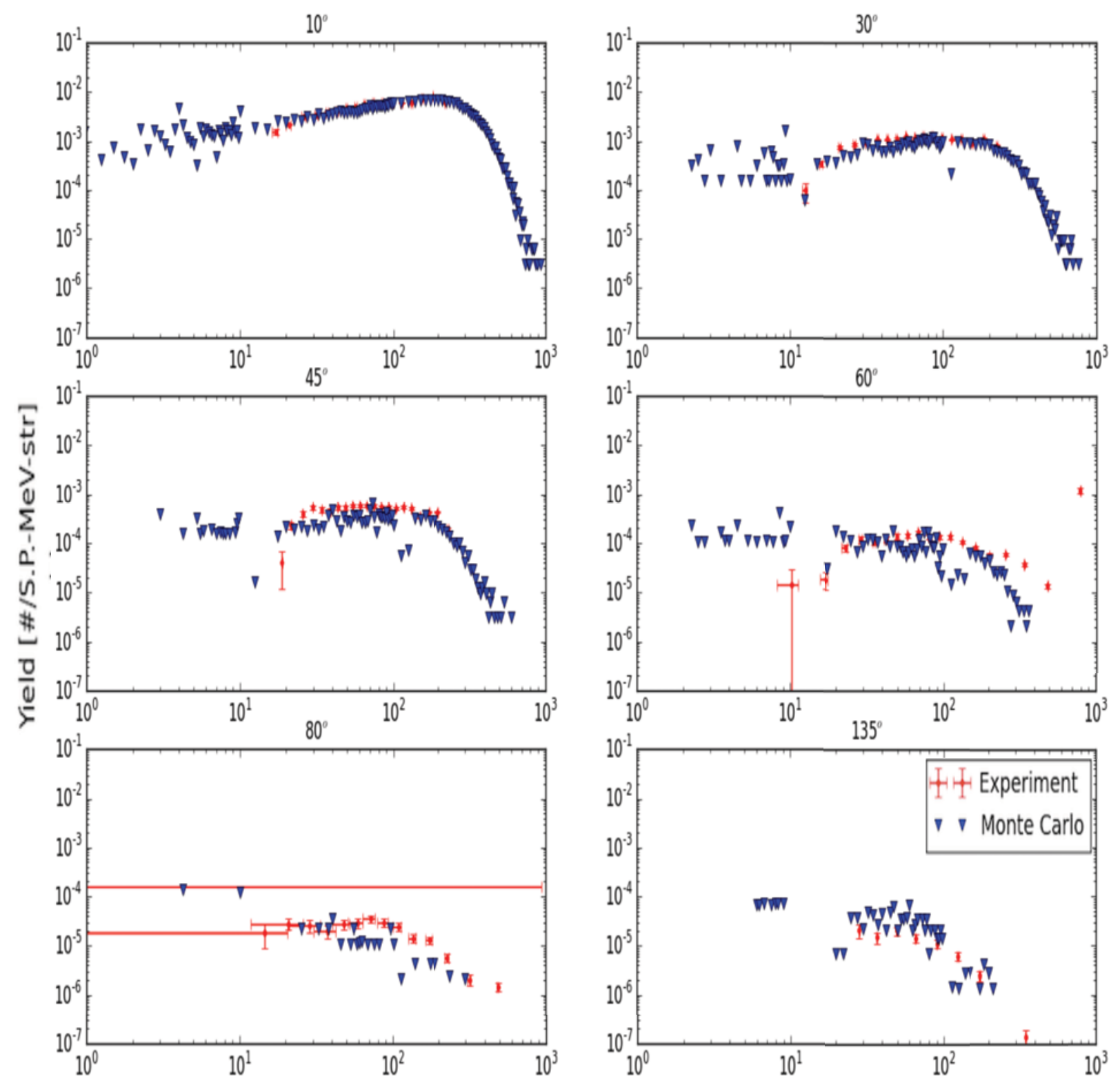

Energy of fragment as it enters detector [MeV]

Fig. 5. Measured and simulated yields of protons resulting form the bombardment of $20 \mathrm{~g} / \mathrm{cm}^{2}$ aluminum upstream target by $0.4-$ $\mathrm{AGeV}$ Fe. 
The $0.4-\mathrm{AGeV}$ iron experiment runs saw approximately 610 million projectile ions delivered with a $64 \%$ live acquisition time. This data rate produced proton yields with statistical uncertainties less than $10 \%$ for angles up to $60^{\circ}$ save for a few low energy (less than 10 $\mathrm{MeV} /$ nucleon) bins. The wider angle detectors had uncertainties generally less than $20 \%$.

MCNP6 simulation results are presented in this work. Other Monte Carlo tools will be utilized to benchmark these results in the future. Physics models selected to adhere to the default and suggested options as published in the software literature. Iron projectile simulations used $1.00 \times 10^{7}$ source particle histories and generated results with statistical errors similar to those of the measured yields. Currently the measured yields agree most closely with the forward-most angle simulation results, differing at most by a factor of two. The spectra shape of these tend to agree, as to where the drop-off in yield as a function of energy should occur, giving confidence in the agreement.

\section{Summary}

Double differential yields of secondary light ions produced by protons, helium and iron in thick targets of aluminium of 20,40 , and $60 \mathrm{~g} / \mathrm{cm}^{2}$ thickness were obtained from accelerator-based measurements performed at NSRL in March 2016. This data will supplement the database of measured ion fluences produced by space-flight probes. Ultimately, this information will help validate transport calculations of GCR through shielding materials used in the assessment of cancer risks associated with prolonged exposures that will occur in missions beyond Low Earth Orbit. These calculations are imperative in safely accomplishing the missions of furthering manned space flight.

\section{Acknowledgements}

This work was supported by Grants NNX12AL51A and NNX15AD89A from the United States National Aeronautics and Space Administration.

\section{References}

1. F. A. Cucinotta, M.-H. Y. Kim, L. Ren, Evaluating shielding effectiveness for reducing space radiation cancer risks, Radiat. Meas., 41, 1173-1185 (2006)

2. T. C. Slaba, X. Xu, S. R. Blattnig, R. B. Norman, GCR environmental models III: GCR model validation and propagated uncertainties in effective dose, Space Weather, 12, 233-245 (2014)

3. T. C. Slaba, S. R. Blattnig, GCR environmental models I: Sensitivity analysis for GCR environments, Space Weather, 12, 217-224 (2014)

4. T. C. Slaba, C. J. Mertens, S. R. Blattnig, Radiation Shielding Optimization on Mars, NASA Technical Paper 2013- 217983 (2013)

5. PSTAR Stopping Power and Range Tables for Protons," National Institute of Standards and
Technology (NIST): Physical Measurements Laboratory. [Online] Available: http://physics.nist.gov/PhysRefData/Star/Text/PSTA $\underline{\text { R.html }}$

6. T. Goorley, et al., Initial MCNP6 Release Overview, Nuclear Technology, 180, 298-315 (2012). 